\title{
A novel coarse-to-fine remote sensing image retrieval system in JPEG-2000 compressed domain
}

\section{Akshara Preethy Byju, Begüm Demir, Lorenzo Bruzzone}

Akshara Preethy Byju, Begüm Demir, Lorenzo Bruzzone, "A novel coarse-tofine remote sensing image retrieval system in JPEG-2000 compressed domain," Proc. SPIE 10789, Image and Signal Processing for Remote Sensing XXIV, 107890T (9 October 2018); doi: 10.1117/12.2327051 


\title{
A Novel Coarse to Fine Remote Sensing Image Retrieval System in JPEG 2000 Compressed Domain
}

\author{
Akshara Preethy Byju ${ }^{1}$, Begum Demir ${ }^{2}$, and Lorenzo Bruzzone ${ }^{1}$ \\ ${ }^{1}$ University of Trento, Via Sommarive, 18 I-38123, Povo, Trento, Italy \\ ${ }^{2}$ Technische Universitat Berlin, Einsteinufer 17, 10587, Berlin, Germany
}

\begin{abstract}
This paper presents a novel content-based image search and retrieval (CBIR) system that achieves coarse to fine remote sensing (RS) image description and retrieval in JPEG 2000 compressed domain. The proposed system initially: i) decodes the code-streams associated to the coarse (i.e., the lowest) wavelet resolution, and ii) discards the most irrelevant images to the query image that are selected based on the similarities estimated among the coarse resolution features of the query image and those of the archive images. Then, the codestreams associated to the sub-sequent resolution of the remaining images in the archive are decoded and the most irrelevant images are selected by considering the features associated to both resolutions. This is achieved by estimating the similarities between the query image and remaining images by giving higher weights to the features associated to the finer resolution while assigning lower weights to those related to the coarse resolution. To this end, the pyramid match kernel similarity measure is exploited. These processes are iterated until the code-streams associated to the highest wavelet resolution are decoded only for a very small set of images. By this way, the proposed system exploits a multiresolution and hierarchical feature space and accomplish an adaptive RS CBIR with significantly reduced retrieval time. Experimental results obtained on an archive of aerial images confirm the effectiveness of the proposed system in terms of retrieval accuracy and time when compared to the standard CBIR systems.
\end{abstract}

Keywords: image retrieval in compressed domain, JPEG 2000 algorithm, coarse to fine image retrieval, remote sensing.

\section{INTRODUCTION}

Due to the continuous advances in satellite technology, recent years have witnessed an explosive growth of remote sensing (RS) image archives. Accordingly, fast and accurate content based image search and retrieval (CBIR) has attracted increasing attention in RS, aiming to seek the most similar images to a query image from large-scale archives [1]. Conventional RS image retrieval techniques often relies on the keywords/metadata associated with the images. However, in the real scenario where terabytes (TB) of data are acquired per day, manual assignment of keywords/metadata to all the images in a large-scale archive is not feasible. In view of that, very recently, the content of the RS images justified to be an efficient attribute to retrieve images from such massive archives. Thus, in the recent years CBIR gained attention in the RS society [1, 2]. A CBIR system generally has two main modules: 1) feature extraction, where each image in an archive is represented using a descriptor; and 2) image matching, where the query image descriptor is matched with all the other image descriptors in the archive. In RS literature, several low-level image descriptors such as texture, color and shape are introduced to characterize the content of the images [3]. To improve the retrieval performance, global feature descriptors obtained from the local regions of an image are found effective. In addition, several region based image retrieval techniques are introduced to model the spatial relationship between the images [2]. For image matching several measures such as histogram intersection (HI), chi-square, earth mover's distances are widely used.

Further author information: (Send correspondence to Akshara Preethy Byju)

Akshara Preethy Byju, e-mail: akshara.preethybyju@unitn.it

Begum Demir, e-mail: demir@tu-berlin.de

Lorenzo Bruzzone, email: lorenzo.bruzzone@unitn.it

Image and Signal Processing for Remote Sensing XXIV, edited by Lorenzo Bruzzone,

Francesca Bovolo, Proc. of SPIE Vol. 10789, 107890T · (C) 2018 SPIE

CCC code: $0277-786 \mathrm{X} / 18 / \$ 18 \cdot$ doi: $10.1117 / 12.2327051$

Proc. of SPIE Vol. 10789 107890T-1 
RS images are usually stored in compressed format in order to reduce the storage size of the archives [4] and thus existing RS CBIR systems require decoding images before applying any feature extraction algorithms (which cannot be directly applied to compressed streams of RS images). However, this process is computationallydemanding and impractical in the case of large-scale RS image retrieval problems. Several compression algorithms are introduced in the RS literature, such as Differential Pulse Code Modulation (DPCM), Adaptive DPCM (ADPCM), Consultative Committee for Space Data Systems-Lossless Data Compression (CCSDS-LDC), Joint Photographic Experts Group (JPEG), lossy and lossless JPEG and JPEG 2000. Among several compression methods, JPEG 2000 has become very popular in RS due to its inherent multiresolution paradigm and it is based on: i) applying successive dyadic discrete wavelet decompositions to the image; and ii) partitioning the wavelet sub-band into code-blocks that are then entropy-coded into an embedded bit-stream independently [5].

In computer vision and pattern recognition, several approaches were introduced to obtain features from the JPEG 2000 compressed images. When JPEG 2000 is considered two types of descriptors can be considered: i) header descriptors, where the descriptors are directly obtained from the fully compressed bit stream; and ii) wavelet descriptors, where the descriptors that are obtained from a partially decoded (entropy-decoded) wavelet coefficients [6]. Header descriptors include: i) the number of bytes used to entropy encode image code-blocks; and ii) the number of significant bit planes of a code-block. Obtaining discriminant features from the partially decoded wavelet coefficients have been an active research area in computer vision. Several studies are performed on the various possibilities to extract texture, shape and color descriptors from the wavelet coefficients $[7,8]$ (which requires entropy decoding process applied to all images in the archives). One among the earliest studies is to calculate the mean and variance of each sub bands at different resolutions and compare it with the descriptor obtained from the query image $[9,10]$. Further, several studies are also performed to exploit the near-Gaussian behavior of the high-frequency sub bands [11, 12].

To address the above-mentioned limitations in the existing RS CBIR systems, in this paper we present a novel coarse to fine image retrieval system that aims at retrieving images by minimizing the amount of decompressed data without reducing the retrieval performance. The proposed retrieval system begins with features extracted in the coarse resolution and then discards the most irrelevant images to the query image based on the similarities estimated among the coarse resolution features of the query image and those of the archive images. Then, codestreams associated to the sub-sequent resolution (higher resolution) are decoded only for the remaining images (which are more relevant to the query image) and the features associated to this resolution are extracted. By this way, each (non-discarded) image is represented by increasingly fine descriptors associated to the first two resolutions. Then the similarities between the query image and these images are computed by giving higher weights to the features associated to the finer resolution while assigning lower weights to those related to the coarse resolution. This is achieved by using the pyramid match kernel similarity measure presented in [13]. Then, the most irrelevant images are discarded and these processes are iterated until the code-streams associated to the highest wavelet resolution is decoded only for a very small set of images (those are highly relevant to the query image). By this way, the proposed system exploits a multiresolution and hierarchical feature space and accomplish an adaptive RS CBIR with an optimal resource usage in terms of retrieval time. It is worth noting that for each resolution level the same feature extraction method is considered (i.e., the type of the features remains the same), whereas the features associated to the finer resolutions are computed for a smaller set of the archive images. Experimental results were obtained in a benchmark archive exhibit the effectiveness of the proposed retrieval system.

The remainder of this paper is organized as follows: Section 2 presents an overview of the JPEG 2000 algorithm where Section 3 presents the proposed system. Section 4 details the considered archive and the experimental results performed. Finally, Section 5 draws the conclusion of the presented work.

\section{JPEG 2000 OVERVIEW}

Figure 1 shows an overview of the JPEG 2000 compression algorithm [5]. Initially the input image is split into several small non-overlapping rectangular blocks called tiles. Each tile from each spectral band of an image is then transformed using Discrete Wavelet Transform (DWT). Successive dyadic wavelet decomposition transforms each tile component into 3-high pass (high frequency) sub bands and 1-low pass (low frequency) sub band thus, allowing for a multiresolution paradigm within the framework. The information obtained from 


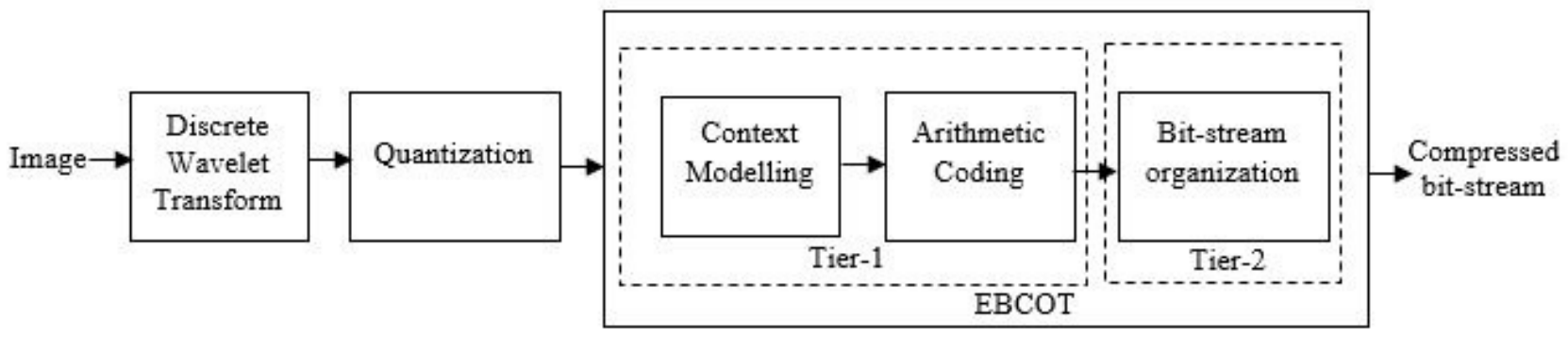

Figure 1. A general scheme of the JPEG 2000 compression algorithm.

successive multiresolution high and low frequency sub bands are utilized in each stage of our proposed coarse to fine CBIR system. JPEG 2000 compression standard supports both lossless as well as lossy coding techniques. Each of these sub bands are further sub-divided into non-overlapping blocks called precincts and each precinct is further divided as code-blocks. Each code-block is usually of the size 32-by-32 or 64-by-64 pixels [5].

Entropy coding is divided into two steps in the standard JPEG 2000 compression algorithm. In Tier1 encoding, each code-block of each component is entropy coded using the Embedded Block Code with Optimal Truncation (EBCOT) that is the coding technique used in JPEG 2000 compression algorithm. For the arithmetic coding, the code blocks are represented using bit planes and these bit planes are coded from Most Significant Bitplane (MSB) to Least Significant Bitplane (LSB) using three coding passes: Significance Propagation pass, Magnitude Refinement pass and clean-up pass. Then, further a context-sensitive binary arithmetic coder encodes the obtained bits obtained after the three coding passes. This is the Tier-1 encoding in JPEG 2000 compression algorithm. Thus, the algorithm supports bit depth scalability to any range which is another added advantage.

In Tier-2 encoding, the bit-stream obtained from the arithmetic coder is organized as packets and layers. Packets contain the bit stream organization of the code blocks and have a packet header that saves the location of the bit stream related to a particular precincts where as layers contain information about all the packets that are arranged into bit streams. Packets and Layers can be arranged using resolution, layer, component or spatial location of the image. This arrangement allows for a progressive encoding as well as decoding order based on multiresolution which is utilized for a hierarchical coarse to fine approach in this work.

\section{PROPOSED COARSE TO FINE RS IMAGE RETRIEVAL SYSTEM}

In this paper, we propose a novel coarse to fine CBIR system to retrieve images from large-scale JPEG 2000 compressed RS image archives. Figure 2 shows the general block scheme of the proposed coarse to fine RS CBIR system. Let $\mathbf{X}=\left\{X_{1}, X_{2}, \ldots, X_{N}\right\}$ be an archive of a very large number $N$ of compressed RS images where $X_{i}$ is the $i$-th image. Given a query image $X_{Q}$, we aim to retrieve a set of images semantically very similar to $X_{Q}$ from the archive. The main objective of the proposed system to retrieve similar images from $\mathbf{X}$ with respect to $X_{Q}$ that share similar attributes. The proposed system initially decodes the code-streams associated to coarse resolution for all the $N$ images in the archive. The features obtained at a coarse level (lowest wavelet resolution) describes global structure or large-scale objects and the features obtained at fine level (higher wavelet resolution) are able to capture local detailed information of fine-scale objects. On the basis of this analysis, the system initially obtains coarser features and discards most irrelevant images with reference to the query image. With the coarser features, the proposed system discards some of the most distinctive $\theta \%$ images from the archive. Then, in the next retrieval stage, only the code-streams of higher wavelet resolution associated with the subset of relevant images obtained from the previous stage is decoded and then again a certain amount of irrelevant image is discarded. The decoding of higher level features and discarding of irrelevant images is iterated until we reach to the highest wavelet resolution. After decoding features at each level, feature matching is performed such that a higher weight is assigned to finer features that represent local detailed information of the image. As decoding of all the images in the archive is ineffective in terms of cost as well as computational time, through this proposed system we focus only on decoding wavelet decomposition levels of the most relevant images with respect to the 


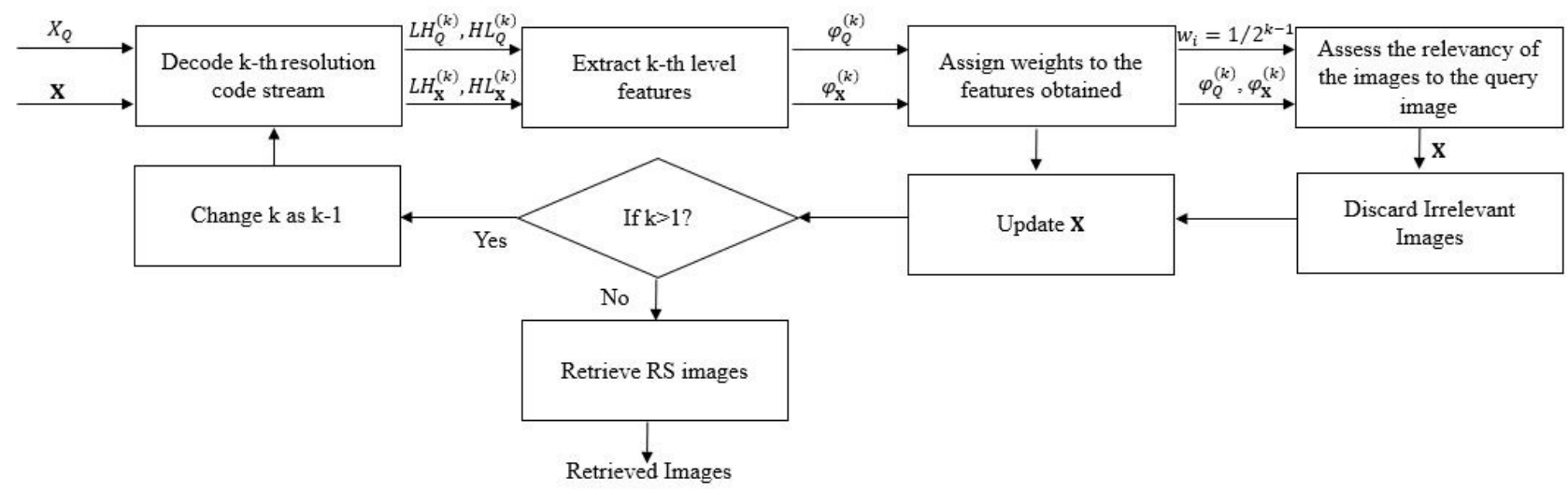

Figure 2. General block scheme of the proposed coarse to fine RS CBIR system in the compressed domain.

query image. In this manner, the proposed system exploits a multiresolution and hierarchical feature space and accomplish an adaptive RS CBIR system with an optimal resource usage in terms of the retrieval time. The subsections below details about the multiresolution descriptors obtained as well as the hierarchical weighting approach adopted for the proposed system.

\subsection{Hierarchical multiresolution descriptors}

At each wavelet decomposition level, hierarchical multiresolution features are obtained from the partially decoded code streams of the JPEG 2000 compressed image in the archive to match with that of $X_{Q}$. Let us consider that the images in the archive are represented up to $k$ wavelet decomposition levels. Thus, an image has $3 k$ high pass sub bands and $k$ low pass sub bands. Thus, at each level $k$ for each image component we obtain approximation $\left(L L^{(k)}\right)$, horizontal $\left(L H^{(k)}\right)$, vertical $\left.\left(H L^{(k)}\right)\right)$ and diagonal $\left(H H^{(k)}\right)$ sub bands of size $m$ by $n$. Inspired from the work presented by [14], we represent the moduli of the horizontal and vertical sub band distribution as a simple descriptor. The moduli of the horizontal and vertical sub band are obtained as:

$$
\varphi_{m, n}^{(k)}=\sqrt{\left(L H_{m, n}^{k}\right)^{2}+\left(H L_{m, n}^{\mathbf{k}}\right)^{2}}
$$

where, $\varphi^{(k)}$ is the moduli distribution obtained at any decomposition level $k$. Let us assume that there exist a positive integer $p$ such that the value $\varphi^{(k)} \leq 2^{p}$. Thus, at each decomposition level $k$ of an image, we identify texture descriptor such that $\varphi^{(k)} \in\left[(\mathrm{a}-1) \cdot 2^{p}\right.$, a. $\left.2^{p}\right]$ where $a$ represents the bin-width. Then, we represent the number of $\varphi^{(k)}$ obtained at each decomposition level using a histogram with $p$ number of bins. Finally, one may obtain the normalized histogram texture descriptor obtained for image $i$ at decomposition level $k$. The descriptor obtained at level $k$ of all the components are merged together to represent the texture information obtained from that resolution. It is worth noting that, the decoding step as well as the feature weight assigning steps appropriately conform with the JPEG 2000 decoding technique.

\subsection{Weighted coarse to fine image retrieval using pyramid match kernel}

Inspired from the work presented by Grauman and Darrell [13], a pyramid match kernel similarity measure that estimate the similarity between multiresolution histogram is adopted to hierarchically assign weight to the coarse to fine multiresolution features that were obtained from the previous step. Here, we consider the inherent correspondences between the descriptor of query image $X_{Q}$ at level $k$ is matched with that of the archive images. The descriptors obtained from the coarser resolutions of an image is able to capture the global texture information in an image while the features obtained from fine resolutions can attain the local detailed texture information. Let $X_{I}$ be any image from the archive, then we obtain the pyramid match similarity between $X_{Q}$ and $X_{I}$ as:

$$
\operatorname{PMK}\left(\left(X_{Q}\right),\left(X_{I}\right)\right)=\sum_{i=m}^{k} w_{i} \cdot N_{i}, m<k
$$


where $w_{i}=\frac{1}{2^{i-1}}$ is the weight assigned to each of the multiresolution features and $N_{i}$ is the number of inherent correspondence between $X_{Q}$ and $X_{I}$ at any decomposition level $i$. Here, the new implicit correspondences between $X_{Q}$ and $X_{I}$ is obtained as;

$$
N_{i}=H I\left(H_{Q}^{k}-H_{I}^{k}\right)-H I\left(H_{Q}^{(k-1)}-H_{I}^{(k-1)}\right)
$$

where $H I$ is the histogram intersection distance between the descriptors associated to the coarser decomposition level $k$ and a finer decomposition level $k-1$ performed to obtain inherent correspondence between $X_{Q}$ and $X_{I}$ at any level $k$. It is worth noting that in this step of the proposed CBIR system weight is assigned considering the fact the finer features are more discriminant with respect to the coarser features. Assigning $w_{i}$ to the progressively decoded features is providing higher weights to more discriminant JPEG 2000 decoded feature further minimizing the amount of irrelevant images in the further retrieval process.

\section{EXPERIMENTAL RESULTS}

To assess the effectiveness of the proposed compressed domain RS CBIR system, the well-known UCMERCED benchmark archive broadly categorized into 21 classes with 100 images each is used (see Table 1). Each image in the archive is of size 256 by 256 pixels with $0.3 \mathrm{~m}$ spatial resolution. The images are categorized into class labels as: agricultural, airplane, baseball diamond, beach, buildings, chaparral, dense residential, forest, freeway, golf course, harbor, intersection, medium residential, mobile home park, overpass, parking lot, river, runway, sparse residential, storage and tennis court. To assess the performance of the proposed method, we have used the multi-label annotations of images that are described in [2] and available at 'http://bigearth.eu/datasets'. The total number of considered primitive class labels is 17 (which are: airplane; bare-soil; buildings; cars; chaparral; court; dock; field; grass; mobile-home; pavement; sand; sea; ship; tanks; trees; water), while the number of labels associated with each image varies between 1 and 7. The UCMERCED archive [15] has three image component and is available in .tif format. For the experimental purposes, all the images in the archive is compressed using JPEG 2000 algorithm. In our experiments, a three-level wavelet decomposition is adopted due to the size constraint of the images in the archive. The minimum size of the code-block of a JPEG 2000 compressed image must be 32-by-32 or 64-by-64 [5]. A 32-by-32 code-block size is attained after three-level wavelet decomposition $(k=3)$ which also allows for a coarse to fine retrieval system. To analyze the performance of the proposed compressed domain RS CBIR system, recall and precision metrics used for single-label image retrieval cannot be considered. Recall and precision for multi-label image retrieval is used to analyze the performance of the proposed RS CBIR system. Let $X_{R}$ be the number of images retrieved from the proposed RS CBIR system. In the experiments, we retrieved first 20 ranked images based on the pyramid match kernel similarity measure. Let $L_{Q}$ and $L_{I}$ be the multi-labels associated with the query image and the considered image from the archive. The precision and recall can be evaluated as [2]:

$$
\begin{gathered}
\text { Recall : } \frac{1}{\left|X_{R}\right|} \sum_{i=1}^{X_{R}} \frac{L_{Q} \cap L_{I}}{L_{Q}} \\
\text { Precision }: \frac{1}{\left|X_{R}\right|} \sum_{i=1}^{X_{R}} \frac{L_{Q} \cap L_{I}}{L_{I}}
\end{gathered}
$$

In the conventional RS CBIR system, the images require full decoding before performing any retrieval process. In the experiments, we compared the proposed system with: i) a standard CBIR system that fully decompresses whole images before extracting image features; and ii) a CBIR system that partially decompresses the images but does not consider any coarse to fine retrieval approach. Table 1 shows the recall and precision performance metrics evaluated for the traditional RS CBIR system, RS CBIR system without a coarse to fine approach and the proposed system. The average multi-label recall and precision values evaluated for the proposed system are $70.94 \%$ and $68.28 \%$ respectively when $\theta=50 \%$. From Table 1 , one may notice that the recall and precision of the proposed compressed domain coarse to fine retrieval system outperform the conventional RS CBIR system that requires complete decompression. Also, there is an improvement in the average precision and recall measures when compared to a RS CBIR system without coarse to fine approach. In addition, the efficiency of the proposed 


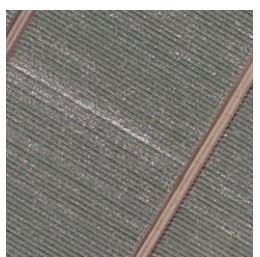

(a) field

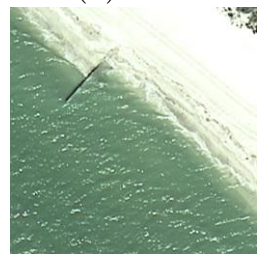

(d) sea, sand

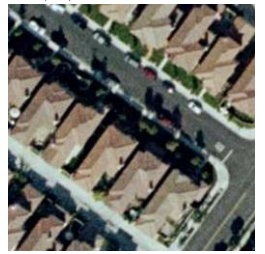

(g) buildings, pavement, cars

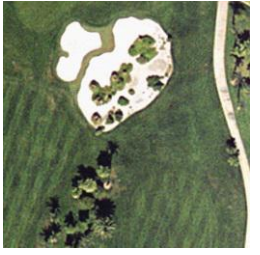

(j) grass, tress, bare-soil

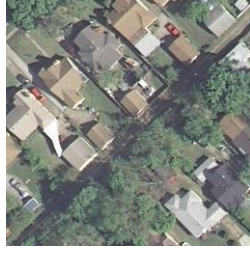

(m) trees, buildings, pavement, cars

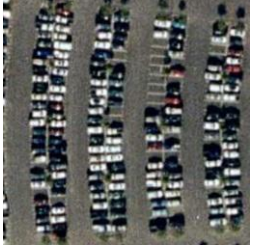

(p) cars, pavement

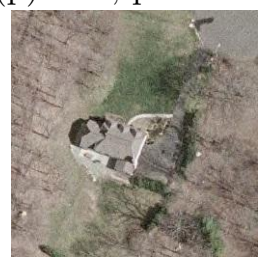

(s) buildings, grass, bare-soil, pavement

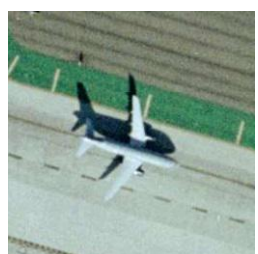

(b) airplane, pavement, grass

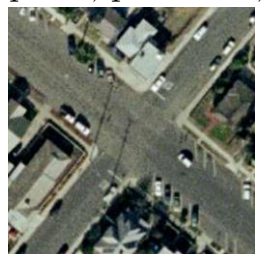

(e) buildings, pavement, cars

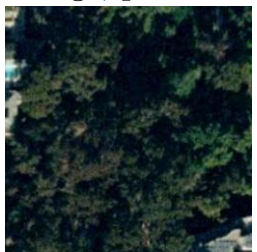

(h) trees, bare-soil

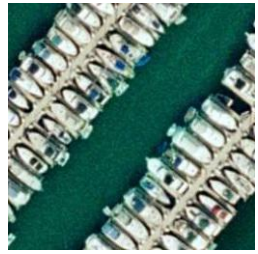

(k) ship, dock, water

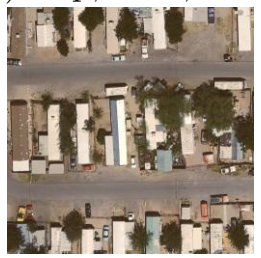

(n) mobile-home, pavement, cars

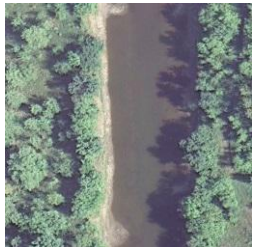

(q) water, trees, bare-soil

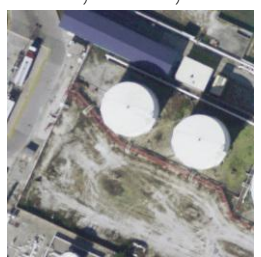

(t) tanks, bare-soil, pavement

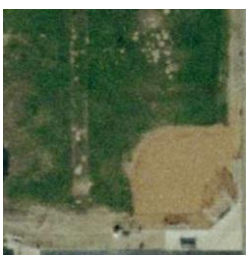

(c) bare-soil, pavement, grass

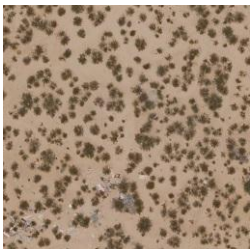

(f) sand,chaparral

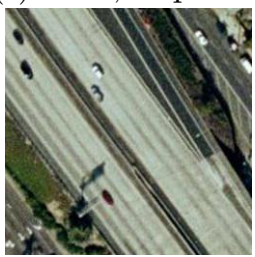

(i) pavement, cars, grass

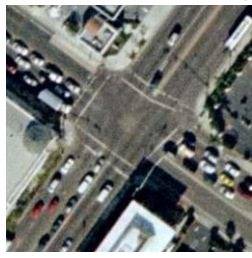

(l) pavement, cars, building

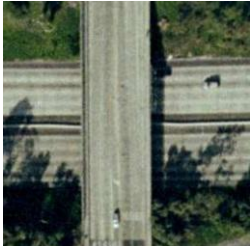

(o) pavement, cars, trees

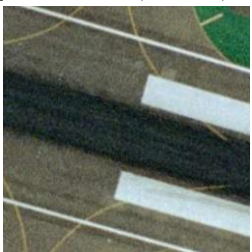

(r) pavement, grass

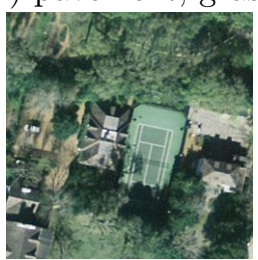

(u) court, grass, trees, buildings

Figure 3. Examples of images in the considered archive and their associated class labels. 
Table 1. Average multi-label recall and precision evaluated for the conventional CBIR, CBIR without coarse to fine and the proposed CBIR.

\begin{tabular}{|c|c|c|}
\hline Method & Precision (\%) & Recall(\%) \\
\hline Conventional RS CBIR system (SIFT features) & 68.73 & 65.68 \\
\hline RS CBIR system without coarse to fine approach & 70.87 & 68.18 \\
\hline Proposed RS CBIR & $\mathbf{7 0 . 9 4}$ & $\mathbf{6 8 . 2 8}$ \\
\hline
\end{tabular}

Table 2. Computational time for the conventional CBIR, the CBIR without coarse to fine and the proposed CBIR.

\begin{tabular}{|c|c|c|}
\hline Method & Decoding time (sec) & Feature extraction time (sec) \\
\hline Conventional RS CBIR system (SIFT features) & 113.65 & 361.50 \\
\hline RS CBIR system without coarse to fine approach & 89.74 & 51.14 \\
\hline Proposed RS CBIR & $\mathbf{5 8 . 1 8}$ & $\mathbf{2 9 . 0 8}$ \\
\hline
\end{tabular}

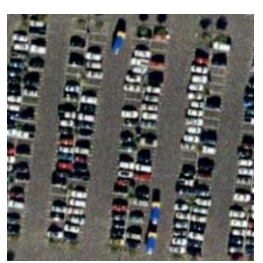

(a)

$1^{s t}$
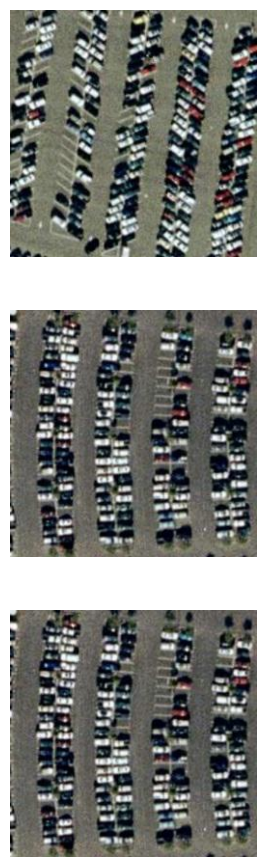

$5^{t h}$
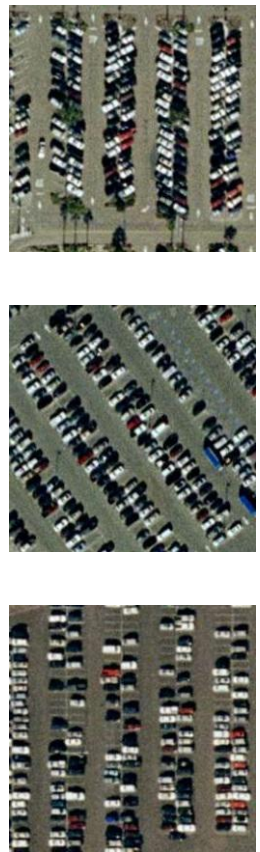

$10^{t h}$

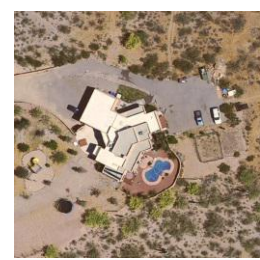

(b)

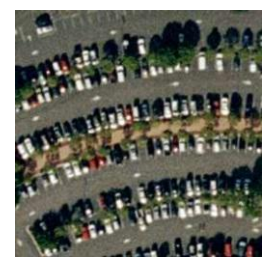

(c)

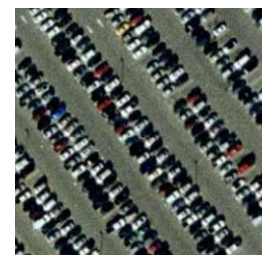

$15^{\text {th }}$
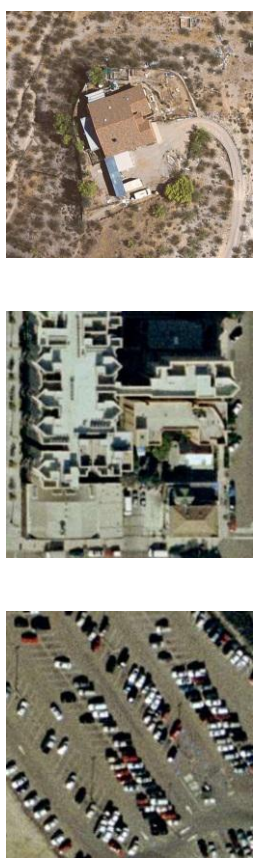

(d)

Figure 4. (a) Query image; (b) retrieved images by the conventional RS CBIR that uses SIFT features; (b) retrieved images by the RS CBIR system without coarse to fine approach; and (c) retrieved images by the proposed RS CBIR system.

system is very well noticed with respect to the images retrieved and the computational time. Figure 4 shows the images retrieved for both the three systems for the given parking lot query image ranked $1^{s t}, 5^{t h}, 10^{t h}$ and $15^{\text {th }}$ for the given proposed compressed domain RS CBIR and conventional RS CBIR system. From Figure 3, one may notice that the classes associated with Parking lot category are bare soil, cars, grass and pavement where as for the Building it is namely cars, buildings and pavement. If we observe the $1^{\text {st }}, 5^{\text {th }}, 10^{\text {th }}$ and $15^{\text {th }}$ 
images of the proposed system it is same to that of the query image. While, if we analyze the retrieved images of the standard CBIR system as well as the RS CBIR system without coarse to fine one may see that the image from the building category is also retrieved. Also, if we analyze the $5^{\text {th }}$ ranked retrieved image of the proposed compressed domain RS CBIR system, we may notice that it has a similar texture pattern as compared to the query image. Although the image at $10^{t h}$ rank belongs to the same category the texture orientation is different whereas the same pattern is visible at $5^{t h}$ rank of the RS CBIR system without a coarse to fine approach. This shows that the weight assigned to the pyramid match similarity kernel has a direct effect on the ranking of the images. As an example, higher weight assigned to finer features are able to identify large-scale objects and can retrieve images of similar pattern as that of the query image. Table 2 compares the computational time required for the proposed compressed domain RS CBIR system and conventional RS CBIR system. It is evident that the time taken to perform the retrieval for the proposed coarse to fine system is less as compared to the others. The feature extraction time required is almost halved $(58.18 \mathrm{sec})$ as compared to the conventional RS CBIR system $(113.65 \mathrm{sec})$. In addition, there is a significant improvement in the feature extraction time (i.e. $29.08 \mathrm{sec}$ ) when compared to the other two systems. The time taken to decode the lowest wavelet resolution for all the $N$ images in the archive is less than the time taken to decode $N-\theta$ images from a highest wavelet resolution. This is utilized to improve the computational time while decoding the features at each level with less number of images. Discarding irrelevant images at each stage makes the proposed system less time-demanding as compared to the existing image retrieval systems.

\section{CONCLUSION}

In this paper we introduced a novel content-based image search and retrieval (CBIR) system that attains a coarse to fine remote sensing (RS) image description and retrieval in JPEG 2000 compressed domain. The proposed system considers that the amount of data that need to be entropy decoded is directly related to the importance of the image in the retrieval process and thus it aims at searching and retrieving images by minimizing the amount of decompressed data without reducing the retrieval performance. The proposed image retrieval system initially decodes the code-streams associated with the lowest wavelet resolution to extract coarse features and then discards a certain amount of irrelevant images from the archive. We further decode the subsequent resolution code-streams associated with the subset of remaining relevant images and again discard some of irrelevant images. Thanks to the pyramid match kernel measure that hierarchically assign weights to the coarse to fine approach considering the fact that, the features obtained from finer resolution has more discriminative power as compared to the coarser resolution is utilized in a coarse to fine approach in our proposed system. Finally, using the features obtained from the highest wavelet resolution (finest features) we perform image retrieval using the small subset of highly relevant images obtained from the previous stages. Discarding images after decoding each multiresolution feature is further speeding up the retrieval rate as the decoding of further features depends on the relevance of the image with the given query image. Experimental results obtained on a benchmark archive show that the proposed system results in similar accuracies with respect to the standard CBIR system with significantly reduced retrieval time. As a future development, we plan to develop deep networks that can model the images and apply retrieval in compressed domain.

\section{References}

[1] Chaudhuri, B., Demir, B., Chaudhuri, S., and Bruzzone, L., "Multilabel Remote Sensing Image Retrieval Using a Semisupervised Graph-Theoretic Method," IEEE Transactions on Geoscience and Remote Sensing 56(2), 1144-1158 (2018).

[2] Chaudhuri, B., Demir, B., Member, S., and Bruzzone, L., "Region-Based Retrieval of Remote Sensing Images Using an Unsupervised Graph-Theoretic Approach," IEEE Transactions on GeoScience and Remote Sensing Letters 13(7), 987-991 (2016).

[3] Napoletano, P., "Visual descriptors for content-based retrieval of remote sensing images," International Journal of Remote Sensing 39(5), 1343-1376 (2018).

[4] Zhou, S., Deng, C., and Zhao, B., "Remote Sensing Image Compression : A Review," in [IEEE International Conference on Multimedia Big Data], 406-410 (2015). 
[5] Skodras, A., Christopoulos, C., and Ebrahimi, T., "The JPEG 2000 Still Image Compression Standard," IEEE Signal Processing Magazine, 36-58 (2001).

[6] Descampe, A., De Vleeschouwer, C., Vandergheynst, P., and Macq, B., "Scalable feature extraction for coarse-to-fine JPEG 2000 image classification.," IEEE Transactions on Image Processing 20(9), 2636-49 (2011).

[7] Jiang, J., Guo, B. F., and Ipson, S., "Shape-based image retrieval for JPEG-2000 compressed image databases," Multimedia Tools and Applications 29(2), 93-108 (2006).

[8] Dong, Y. and Ma, J., "Wavelet-Based Image Texture Classification Using Local Energy Histograms," IEEE Signal Processing Letters 18(4), 247-250 (2011).

[9] Pi, M. H., Tong, C. S., Choy, S. K., and Zhang, H., "A Fast and Effective Model for Wavelet Subband Histograms and Its Application in Texture Image Retrieval," IEEE Transactions on Image Processing 15(10), 3078-3088 (2006).

[10] Nikam, S. B. and Agarwal, S., "Wavelet energy signature and GLCM features-based fingerprint antispoofing," in [Proceedings of the 2008 International Conference on Wavelet Analysis and Pattern Recognition], 30-31 (2008).

[11] Choy, S. K. and Tong, C. S., "Statistical wavelet subband characterization based on generalized gamma density and its application in texture retrieval," IEEE Transactions on Image Processing 19(2), 281-289 (2010).

[12] Do, M. N. and Vetterli, M., "Wavelet-based texture retrieval using generalized Gaussian density and Kullback-Leibler distance," IEEE Transactions on Image Processing 11(2), 146-158 (2002).

[13] Grauman, K. and Darrell, T., "The Pyramid Match Kernel : Efficient Learning with Sets of Features," Journal of Machine Learning Research 8, 725-760 (2007).

[14] Ves, E. D., Acevedo, D., Ruedin, A., and Benavent, X., "A statistical model for magnitudes and angles of wavelet frame coefficients and its application to texture retrieval," Pattern Recognition 47(9), 2925-2939 (2014).

[15] Yang, Y. and Newsam, S., "Bag-of-Visual-Words and Spatial Extensions for Land-use Classification," in [Proceedings of the 18th SIGSPATIAL International Conference on Advances in Geographic Information Systems - GIS '10], 270-279 (2010). 